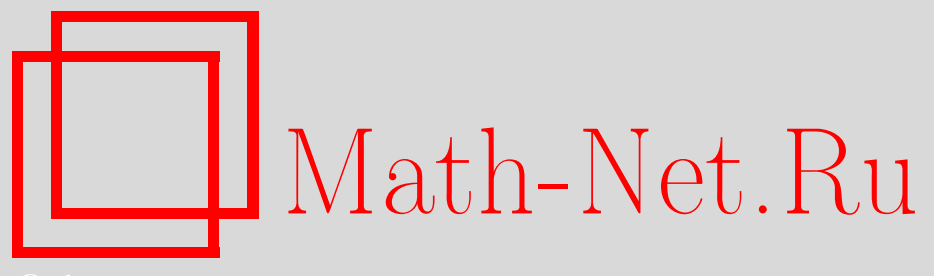

А. А. Куликова, Ю. В. Прохоров, Распределение дробных долей случайных векторов: гауссовский случай. I, Теория вероятн. и ее примен., 2003, том 48, выпуск 2, 399-402

DOI: https://doi.org/10.4213/tvp293

Использование Общероссийского математического портала MathNet.Ru подразумевает, что вы прочитали и согласны с пользовательским соглашением

http://www . mathnet.ru/rus/agreement

Параметры загрузки:

IP: 54.224 .135 .184

26 апреля 2023 г., $14: 37: 35$

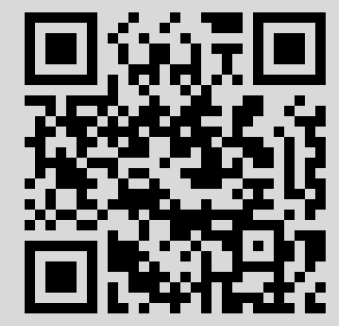


(C) 2003 г.

КУЛИКОВА А. А. ${ }^{*}$, ПРОХОРОВ Ю. В. ${ }^{* *}$

\title{
РАСПРЕДЕЛЕНИЕ ДРОБНЫХ ДОЛЕЙ СЛУЧАЙНЫХ ВЕКТОРОВ: ГАУССОВСКИЙ СЛУЧАЙ. ${ }^{1)}$
}

\begin{abstract}
В статье рассматривается распределение вероятностей дробной части $s$-мерного гауссовского случайного вектора. Доказаны неравенства для отклонения этого распределения от равномерного. Доказательства используют формулу суммирования Пуассона и некоторые факты теории представлений целых чисел квадратичными формами. Основное внимание этой части работы уделено случаю относительно небольших значений $s$. Случай весьма больших значений $s$ будет рассмотрен дополнительно.
\end{abstract}

Ключевые слова и фразы: равномерное распределение, формула суммирования Пуассона, дробные доли гауссовских векторов.

1. Обозначения. Будут использованы следующие обозначения. Если $x=$ $\left(x_{1}, \ldots, x_{s}\right) \in \mathbf{R}^{s}$, то $|x|=\sqrt{x_{1}^{2}+\cdots+x_{s}^{2}} ;$ если $t=\left(t_{1}, \ldots, t_{s}\right) \in \mathbf{R}^{s}$, то $(t, x)=$ $t_{1} x_{1}+\cdots+t_{s} x_{s} ; \mathbf{Z}^{s}$ - совокупность всех точек $\mathbf{R}^{s}$ с целочисленными координатами; $V_{s}=\pi^{s / 2} / \Gamma(s / 2+1)$ - объем единичного шара в $\mathbf{R}^{s} ; f(t ; X)$ - характеристическая функция случайного вектора $X \in \mathbf{R}^{s} ; p(x ; X)$ - соответствующая плотность вероятности (если она существует); если $z \in \mathbf{R}$, то $\{z\}$ - дробная часть числа $z$; если $z=\left(z_{1}, \ldots, z_{s}\right) \in \mathbf{R}^{s}$, то $\{z\}=\left(\left\{z_{1}\right\}, \ldots,\left\{z_{s}\right\}\right) ; r_{s}(N)$ - число целых точек на сфере $|m|^{2}=N$ в $\mathbf{R}^{s} ; \sigma_{k}(N)$ - сумма $k$-х степеней делителей числа $N ; \widetilde{\sigma}_{1}(N)$ - сумма делителей $N$, кратных четырем.

2. Оценка величины $|p(x ;\{\sigma X\})-1|$. Пусть $X$ - гауссовский случайный вектор в пространстве $\mathbf{R}^{s}$ с нулевым средним и матрицей ковариации $\Sigma, \lambda>0$ - минимальное собственное значение $\Sigma$. Характеристическая функция $X$ имеет вид

$$
f(t ; X)=e^{-(\Sigma t, t) / 2} .
$$

Оценим отклонение случайной величины $\{\sigma X\}(\sigma>0)$ от равномерного распределения в кубе $[0,1]^{s}$.

Пользуясь формулой суммирования Пуассона, получаем при $x \in[0,1]^{s}$

$$
\begin{aligned}
p(x ;\{\sigma X\}) & =\sum_{l \in \mathbf{Z}^{s}} p\left(x_{1}+l_{1}, \ldots, x_{s}+l_{s} ; \sigma X\right)=\sum_{m \in \mathbf{Z}^{s}} f(2 \pi m ; \sigma X) e^{-2 \pi i(m, x)} \\
& =1+\sum_{m \in \mathbf{Z}^{s}, m \neq 0} e^{-\left(\sigma^{2} \Sigma(2 \pi m), 2 \pi m\right) / 2} e^{-2 \pi i(m, x)} \\
& =1+\sum_{m \in \mathbf{Z}^{s}, m \neq 0} e^{-2 \pi^{2} \sigma^{2}(\Sigma m, m)} e^{-2 \pi i(m, x)} .
\end{aligned}
$$

Следовательно,

$$
\Delta=\sup _{x \in[0,1]^{s}}|p(x ;\{\sigma X\})-1|=\left|\sum_{m \in \mathbf{Z}^{s}, m \neq 0} e^{-2 \pi^{2} \sigma^{2}(\Sigma m, m)} e^{-2 \pi i(m, x)}\right|
$$

* Московский государственный университет им. М.В. Ломоносова, факультет вычислительной математики и кибернетики, Ленинские горы, 119992 Москва, Россия; e-mail: anna_q@mail.ru

** Математический институт им. В. А. Стеклова РАН, ул. Губкина, 8, 119991 Москва, ГСП-1, Россия.

1) Работа выполнена при частичной поддержке РФФИ (грант № 03-01-00259). 


$$
\begin{aligned}
& \leqslant \sum_{m \in \mathbf{Z}^{s}, m \neq 0}\left|e^{-2 \pi^{2} \sigma^{2}(\Sigma m, m)} e^{-2 \pi i(m, x)}\right|=\sum_{m \in \mathbf{Z}^{s}, m \neq 0} e^{-2 \pi^{2} \sigma^{2}(\Sigma m, m)} \\
& \leqslant \sum_{m \in \mathbf{Z}^{s}, m \neq 0} e^{-2 \pi^{2} \sigma^{2} \lambda|m|^{2}}=\sum_{N=1}^{\infty} \sum_{m:|m|^{2}=N} e^{-2 \pi^{2} \sigma^{2} \lambda N}=\sum_{N=1}^{\infty} r_{s}(N) q^{N},
\end{aligned}
$$

где $q=e^{-2 \pi^{2} \sigma^{2} \lambda}$.

З а м е ч а н и е. Очевидно, что

$$
\Delta \geqslant|p(0 ;\{\sigma X\})-1| \geqslant \sum_{N=1}^{\infty} r_{s}(N) \tilde{q}^{N},
$$

где $\widetilde{q}=e^{-2 \pi^{2} \sigma^{2} \Lambda}, \Lambda-$ максимальное собственное значение $\Sigma$. Отсюда видно, что указанные верхняя и нижняя оценки для $\Delta$ отличаются тем сильнее, чем больше отношение $\Lambda / \lambda$.

3. Случай $s=4$. По [1, теорема 386]

$$
r_{4}(N)=8 \sigma_{1}(N)-\widetilde{\sigma}_{1}(N),
$$

и, следовательно,

$$
r_{4}(N) \leqslant 8 \sigma_{1}(N) .
$$

Тогда, пользуясь $[2,24.3 .3 \mathrm{I} \mathrm{B}]$, получаем

$$
\sum_{N=1}^{\infty} r_{4}(N) q^{N} \leqslant 8 \sum_{N=1}^{\infty} \sigma_{1}(N) q^{N}=8 \sum_{N=1}^{\infty} \frac{N q^{N}}{1-q^{N}} \leqslant \frac{8}{1-q} \sum_{N=1}^{\infty} N q^{N}=\frac{8 q}{(1-q)^{3}} .
$$

Таким образом,

$$
\Delta \leqslant \frac{8}{(1-q)^{3}} q .
$$

4. Случай $s=8$. По [1, ф-ла в начале п. 20.13 , с. 314$] r_{8}(N)=16(-1)^{N} \times$ $\sum_{d \mid N}(-1)^{d} d^{3}$, и, следовательно,

$$
r_{8}(N) \leqslant 16 \sigma_{3}(N) .
$$

Тогда, пользуясь $[2,24.3 .3$ I B], получаем

$$
\begin{aligned}
\sum_{N=1}^{\infty} r_{8}(N) q^{N} & \leqslant 16 \sum_{N=1}^{\infty} \sigma_{3}(N) q^{N}=16 \sum_{N=1}^{\infty} \frac{N^{3} q^{N}}{1-q^{N}} \\
& \leqslant \frac{16}{1-q} \sum_{N=1}^{\infty} N^{3} q^{N}=\frac{16 q\left(1+4 q+q^{2}\right)}{(1-q)^{5}} .
\end{aligned}
$$

Таким образом,

$$
\Delta \leqslant \frac{16\left(1+4 q+q^{2}\right)}{(1-q)^{5}} q .
$$

5. Случай $s=12$. Пусть $N=2^{\gamma} u$, где $u$ - нечетное. Тогда по [3, теорема 6]

$$
r_{12}(N)=8\left(2+(-1)^{N}\right) \frac{10 \cdot 2^{5 \gamma}+21}{31} \sigma_{5}(u)+8 \sum_{x_{1}^{2}+x_{2}^{2}+x_{3}^{2}+x_{4}^{2}=N}\left(x_{1}^{4}-3 x_{1}^{2} x_{2}^{2}\right) .
$$

Если $N$ - нечетное, то $\gamma=0, u=N$ и

$$
8\left(2+(-1)^{N}\right) \frac{10 \cdot 2^{5 \gamma}+21}{31} \sigma_{5}(u)=8 \sigma_{5}(N)
$$

если $N$ - четное, то $\gamma \geqslant 1$ и

$$
8\left(2+(-1)^{N}\right) \frac{10 \cdot 2^{5 \gamma}+21}{31} \sigma_{5}(u)=24 \frac{10 \cdot 2^{5 \gamma}+21}{31} \frac{2^{5}-1}{2^{5(\gamma+1)}-1} \sigma_{5}(N) \leqslant 8 \sigma_{5}(N) .
$$


Для любого натурального $N$

$$
\begin{aligned}
& 8 \sum_{x_{1}^{2}+x_{2}^{2}+x_{3}^{2}+x_{4}^{2}=N}\left(x_{1}^{4}-3 x_{1}^{2} x_{2}^{2}\right)=2 \sum_{x_{1}^{2}+x_{2}^{2}+x_{3}^{2}+x_{4}^{2}=N}\left(2\left(x_{1}^{4}+x_{2}^{4}+x_{3}^{4}+x_{4}^{4}\right)-N^{2}\right) \\
& \quad \leqslant 2 \sum_{x_{1}^{2}+x_{2}^{2}+x_{3}^{2}+x_{4}^{2}=N}\left(2 N^{2}-N^{2}\right)=2 N^{2} r_{4}(N) \leqslant 16 N^{2} \sigma_{1}(N) .
\end{aligned}
$$

Следовательно, $r_{12}(N) \leqslant 8 \sigma_{5}(N)+16 N^{2} \sigma_{1}(N)$ и

$$
\sum_{N=1}^{\infty} r_{12}(N) q^{N} \leqslant 8 \sum_{N=1}^{\infty} \sigma_{5}(N) q^{N}+16 \sum_{N=1}^{\infty} N^{2} \sigma_{1}(N) q^{N} .
$$

Пользуясь $[2,24.3 .3$ I В], получаем

$$
8 \sum_{N=1}^{\infty} \sigma_{5}(N) q^{N}=8 \sum_{N=1}^{\infty} \frac{N^{5} q^{N}}{1-q^{N}} \leqslant \frac{8}{1-q} \sum_{N=1}^{\infty} N^{5} q^{N}=\frac{8 q\left(1+26 q+66 q^{2}+26 q^{3}+q^{4}\right)}{(1-q)^{7}}
$$

и

$$
\begin{aligned}
16 \sum_{N=1}^{\infty} N^{2} \sigma_{1}(N) q^{N} & =16 \sum_{N=1}^{\infty} \frac{N^{3} q^{N}\left(1+q^{N}\right)}{\left(1-q^{N}\right)^{3}} \leqslant \frac{16}{(1-q)^{3}}\left(\sum_{N=1}^{\infty} N^{3} q^{N}+\sum_{N=1}^{\infty} N^{3} q^{2 N}\right) \\
& =\frac{16}{(1-q)^{3}}\left(\frac{q\left(1+4 q+q^{2}\right)}{(1-q)^{4}}+\frac{q^{2}\left(1+4 q^{2}+q^{4}\right)}{\left(1-q^{2}\right)^{4}}\right) .
\end{aligned}
$$

Таким образом,

$$
\Delta \leqslant\left(\frac{16}{(1-q)^{3}}\left(\frac{1+4 q+q^{2}}{(1-q)^{4}}+\frac{q+4 q^{3}+q^{5}}{\left(1-q^{2}\right)^{4}}\right)+\frac{8\left(1+26 q+66 q^{2}+26 q^{3}+q^{4}\right)}{(1-q)^{7}}\right) q .
$$

6. Случай произвольного $s$. Применяя преобразование Абеля, получаем

$$
\begin{aligned}
\sum_{N=1}^{\infty} r_{s}(N) q^{N} & =\sum_{N=1}^{\infty}\left(r_{s}(1)+\cdots+r_{s}(N)\right)\left(q^{N}-q^{N+1}\right) \\
& =q(1-q) \sum_{N=1}^{\infty}\left(r_{s}(1)+\cdots+r_{s}(N)\right) q^{N-1}
\end{aligned}
$$

Имеем $r_{s}(1)+\cdots+r_{s}(N) \leqslant V_{s}(\sqrt{N}+\sqrt{s} / 2)^{s} \leqslant V_{s}(1+\sqrt{s} / 2)^{s} N^{s / 2}$. Далее,

$$
\sum_{N=1}^{\infty} N^{s / 2} q^{N-1}=1+\sum_{N=1}^{\infty}(N+1)^{s / 2} q^{N} \leqslant 1+2^{s / 2} \sum_{N=1}^{\infty} N^{s / 2} q^{N}
$$

При $y \geqslant 0$ функция $y^{s / 2} q^{y}$ достигает единственного максимума в точке $y_{1}=$ $s /\left(4 \pi^{2} \sigma^{2} \lambda\right)$. Тогда

$$
\sum_{N=1}^{\infty} N^{s / 2} q^{N} \leqslant \int_{0}^{\infty} y^{s / 2} q^{y} d y+2 y_{1}^{s / 2} q^{y_{1}}=\frac{\Gamma(s / 2+1)}{\left(2 \pi^{2} \sigma^{2} \lambda\right)^{s / 2+1}}+2\left(\frac{s}{e 4 \pi^{2} \sigma^{2} \lambda}\right)^{s / 2} .
$$

Следовательно,

$$
\sum_{N=1}^{\infty} N^{s / 2} q^{N-1} \leqslant 1+2^{s / 2}\left(\frac{\Gamma(s / 2+1)}{\left(2 \pi^{2} \sigma^{2} \lambda\right)^{s / 2+1}}+2\left(\frac{s}{e 4 \pi^{2} \sigma^{2} \lambda}\right)^{s / 2}\right) .
$$

Таким образом,

$$
\Delta \leqslant(1-q) V_{s}\left(1+\frac{\sqrt{s}}{2}\right)^{s}\left(1+2^{s / 2}\left(\frac{\Gamma(s / 2+1)}{\left(2 \pi^{2} \sigma^{2} \lambda\right)^{s / 2+1}}+2\left(\frac{s}{e 4 \pi^{2} \sigma^{2} \lambda}\right)^{s / 2}\right)\right) q .
$$


7. Пример. Пусть $X_{n}, n \in \mathbf{Z},-$ гауссовская марковская последовательность. Предположим, что $\mathbf{E} X_{n}=0, \mathbf{D} X_{n}=1$ и коэффициент корреляции $0<\mu<1$. Образуем $s$-мерный гауссовский вектор $X=\left(X_{1}, \ldots, X_{s}\right)$ из первых $s$ членов последовательности. Тогда матрица ковариации $\Sigma$ имеет вид $\Sigma_{i, j}=\mu^{|i-j|}, 1 \leqslant i, j \leqslant s$. Обозначим $\lambda$ - минимальное собственное значение $\Sigma, \Lambda$ - максимальное собственное значение $\Sigma$.

\section{Утверждение.}

$$
\lambda \geqslant \frac{1-\mu}{1+\mu}, \quad \Lambda \leqslant \frac{1+\mu}{1-\mu} .
$$

3 а м е ч а н и е. Этот пример интересен тем, что указанные выше границы для $\lambda$ и $\Lambda$ не зависят от $s$.

Д ок а з а т е в с т в о. По $[4$, с. 7$]$

$$
\Sigma^{-1}\left(y_{1}, \ldots, y_{s}\right)=\frac{1}{1-\mu^{2}}\left(\sum_{j=1}^{s} y_{j}^{2}+\mu^{2} \sum_{j=2}^{s-1} y_{j}^{2}-2 \mu \sum_{j=1}^{s-1} y_{j} y_{j+1}\right) .
$$

По неравенству Коши-Буняковского

$$
\left(\sum_{j=1}^{s-1} y_{j} y_{j+1}\right)^{2} \leqslant\left(\sum_{j=1}^{s} y_{j}^{2}-y_{1}^{2}\right)\left(\sum_{j=1}^{s} y_{j}^{2}-y_{s}^{2}\right) .
$$

На единичной сфере из соотношений

$$
\sum_{j=1}^{s} y_{j}^{2}=1, \quad \sum_{j=2}^{s-1} y_{j}^{2}=1-y_{1}^{2}-y_{s}^{2}, \quad\left|\sum_{j=1}^{s-1} y_{j} y_{j+1}\right| \leqslant \sqrt{\left(1-y_{1}^{2}\right)\left(1-y_{s}^{2}\right)}
$$

следует

$$
\begin{aligned}
& \frac{1}{1-\mu^{2}}\left(1+\mu^{2}\left(1-y_{1}^{2}-y_{s}^{2}\right)-2 \mu \sqrt{\left(1-y_{1}^{2}\right)\left(1-y_{s}^{2}\right)}\right) \leqslant \Sigma^{-1}\left(y_{1}, \ldots, y_{s}\right) \\
& \leqslant \frac{1}{1-\mu^{2}}\left(1+\mu^{2}\left(1-y_{1}^{2}-y_{s}^{2}\right)+2 \mu \sqrt{\left(1-y_{1}^{2}\right)\left(1-y_{s}^{2}\right)}\right) .
\end{aligned}
$$

Находя максимум функции $1+\mu^{2}\left(1-z_{1}-z_{2}\right)+2 \mu \sqrt{\left(1-z_{1}\right)\left(1-z_{2}\right)}$ и минимум функции $1+\mu^{2}\left(1-z_{1}-z_{2}\right)-2 \mu \sqrt{\left(1-z_{1}\right)\left(1-z_{2}\right)}$ в области $z_{1}, z_{2} \geqslant 0, z_{1}+z_{2} \leqslant 1$, получаем, что

$$
\frac{(1-\mu)^{2}}{\left(1-\mu^{2}\right)} \leqslant \Sigma^{-1}\left(y_{1}, \ldots, y_{s}\right) \leqslant \frac{(1+\mu)^{2}}{\left(1-\mu^{2}\right)} \text {. }
$$

Отсюда

$$
\lambda \geqslant \frac{\left(1-\mu^{2}\right)}{(1+\mu)^{2}}=\frac{1-\mu}{1+\mu}, \quad \Lambda \leqslant \frac{\left(1-\mu^{2}\right)}{(1-\mu)^{2}}=\frac{1+\mu}{1-\mu} .
$$

\section{СПИСОК ЛИТЕРАТУРЫ}

1. Hardy G.H., Wright E.M. An Introduction to the Theory of Numbers. Oxford: Clarendon Press, 1960, $421 \mathrm{p}$.

2. Абрамович М., Стиган И. (ред.) Справочник по специальным функциям с формулами, графиками и математическими таблицами. М.: Наука, 1979, 830 с.

3. Ломадзе Г. А. О представлении чисел суммами квадратов. - Труды Тбил. матем. ин-та им. А. М. Размадзе, 1948, т. 16, с. 273-275.

4. Линник Ю. В. Математическая статистика. Л.: Наука, 1982, 284 с. 